\title{
ON THE MONODROMY GROUPS OF LIFTED EULER EQUATIONS
}

\author{
by KATHRYN KUIKEN and JOHN T. MASTERSON
}

(Received 4 August, 1982)

In [13], Poincaré asked the following question: Which abstract groups can appear as monodromy groups [14] of second order, linear, homogeneous differential equations with meromorphic coefficients (which might depend on one or more parameters) on $\hat{\mathbb{C}}$ ? In the present paper, we initiate a classification of monodromy groups of differential equations on compact Riemann surfaces of genus 1 . We proceed as follows: Let

$$
y^{\prime \prime}+\frac{\alpha}{z} y^{\prime}+\frac{\beta}{z^{2}} y=0 \quad \text { with } \quad \alpha, \beta \in \mathbb{C}
$$

be the general Euler equation [1] which has two regular singular points at 0 and $\infty$ in the extended complex plane $\hat{\mathbb{C}}$. Further, let $y_{v}(z)(\nu=1,2)$ be an arbitrary but fixed pair of linearly independent solutions to (1) valid in a neighborhood of some ordinary point. Analytic continuation of each solution along a closed loop $\Lambda$ in $\hat{C}-\{0, \infty\}$, starting and ending at some fixed base point, produces a new solution $y_{\nu, \Lambda}(\nu=1,2)$ which can be expressed as

$$
y_{1, \Lambda}(z)=a_{\nu 1} y_{1}(z)+a_{v 2} y_{2}(z)
$$

where the constants $a_{\nu \mu}(\nu, \mu=1,2)$ in $\mathbb{C}$ depend on the homotopy class $[\Lambda]$ of $\Lambda$. Clearly, $y_{1, \Lambda}(z) / y_{2, \Lambda}(z)=T \circ\left(y_{1}(z) / y_{2}(z)\right)$ where the Möbius transformation $T: w \mapsto$ $\left(a_{11} w+a_{12}\right) /\left(a_{21} w+a_{22}\right)$ depends on $[\Lambda]$. The set of all Möbius transformations $T$ belonging to every possible closed loop $\Lambda$ in $\hat{\mathbb{C}}-\{0, \infty\}$ forms a group $G$, called the monodromy group of the Euler equation (1). $G$ is generated by the Möbius transformation belonging to a simple, closed loop $\Lambda_{0}$ encircling 0 . Hence, $G$ is cyclic.

We will show that substitution of various meromorphic functions $z=g(w)$ into (1) results in equations (henceforth referred to as untransformed lifted Euler equations) which can be transformed to eliminate their first derivative terms to give equations (called transformed lifted Euler equations) having coefficients doubly periodic with respect to an arbitrary lattice [12] $L=\left\langle n \omega_{1}+m \omega_{2}, n, m \in Z \times Z, \operatorname{Im}\left(\omega_{1} / \omega_{2}\right)>0\right\rangle$. Consequently, the transforms can be viewed as equations on an arbitrary complex torus $\mathbb{C} / L$. Thus, each transformed lifted Euler equation has a monodromy group $G^{*}$. $G^{*}$ is also the monodromy group of the untransformed equation because both lifted equations have the same ratio of linearly independent solutions.

The generators for $G^{*}$ are obtained by the analytic continuation of $y_{1}(g(w)) / y_{2}(g(w))$ along simple, closed loops about the singularities of the transformed equation as well as along any two generating loops for the homotopy group $[\mathbf{1 0}]$ of $\mathbb{C} / L$, where all loops avoid singularities. Equivalently, we find [7] these generators by analytically continuing $y_{1}(z) / y_{2}(z)$ along the images of the above mentioned loops under $g(w)$ in $\hat{\mathbb{C}}$. Our selected

Glasgow Math. J. 25 (1984) 47-57. 
substitutions $g$ will not necessarily be doubly periodic so that these images of loops can be either open arcs or closed loops. These observations will ultimately permit us to determine $G^{*}$ explicitly.

We now proceed with a detailed description of our findings.

THeOREM 1. The substitutions $z=t^{\lambda}(w), \lambda \in \mathbb{C}^{*}$, with $t(w)$ of the form [11]

$$
t(w)=\frac{\sqrt{ }\left(\mathscr{P}(w)-e_{1}\right)+\sqrt{ }\left(\mathscr{P}(w)-e_{2}\right)}{\sqrt{ }\left(e_{2}-e_{1}\right)} \text { with } \quad e_{i}=\mathscr{P}\left(\frac{\omega_{i}}{2}\right), \quad i=1,2
$$

or

$$
t(w)=\frac{\prod_{i=1}^{k} \sigma^{m_{i}}\left(w-a_{i}\right)}{\prod_{j=1}^{l} \sigma^{n_{i}}\left(w-b_{j}\right)} e^{c w} \quad \text { with } \quad k, l>0 ; \quad m_{i}, n_{i} \in Z^{+} ; \quad \sum_{i=1}^{k} m_{i}-\sum_{j=1}^{l} n_{i}=0
$$

into any Euler equation (1) (with difference of indicial roots $r=r_{1}-r_{2}$ ) on $\hat{\mathbb{C}}$ produce lifted Euler equations which can be transformed respectively into

$$
y^{\prime \prime}+\frac{1}{2}\left[\frac{1-(r \lambda)^{2}}{2}\left(\mathscr{P}(w)-e_{3}\right)-\frac{3}{2} \mathscr{P}\left(w-\frac{\omega_{1}+\omega_{2}}{2}\right)\right] y=0 \quad \text { where } \quad e_{3}=\mathscr{P}\left(\frac{\omega_{1}+\omega_{2}}{2}\right)
$$

or

$$
y^{\prime \prime}+\frac{1}{2}\left[\frac{1-(r \lambda)^{2}}{2}\left(\sum_{i=1}^{k} m_{i} \zeta\left(w-a_{i}\right)-\sum_{j=1}^{1} n_{j} \zeta\left(w-b_{j}\right)+c\right)^{2}+\theta_{2} t(w)\right] y=0,
$$

where $\mathscr{P}, \sigma, \zeta$ are the Weierstrass functions [12] for an arbitrary lattice $L$ and $\theta_{2}$ is the Schwarzian derivative operator [4]. Equations (4) and (5) have coefficients doubly periodic for $L$, can be treated as equations on $\mathbb{C} / L$ and determine monodromy groups on $C / L$.

REMARK 1. It has been shown [11] that the mapping (2) represents a specific non-affine, branched projective structure [4] on an arbitrary unpunctured torus and is associated with a Schwarzian differential equation with non-affine monodromy group. Here we use the mapping (2) to construct differential equations on punctured tori as well as to determine the monodromy groups of these equations. Similarly, the mappings of type (3) represent classically known affine structures on unpunctured tori [3] and are used here to construct differential equations on tori with arbitrarily many punctures as well as to find the monodromy groups of these equations.

Proof. The Euler equation (1) with difference of indicial roots $r=\sqrt{ }\left((\alpha-1)^{2}-4 \beta\right)$ (see p. 174-177 of [1] for all relevant facts concerning Euler's equation) can be lifted by the map $z=t^{\lambda}(w)$ to $\mathbb{C}$ by a two step process as follows: Let $z=f \circ t(w)$ with $f(t)=t^{\lambda}$. First, lift (1) by $z=f(t)$ to a new Euler equation

$$
y^{\prime \prime}(t)+\frac{\alpha^{\prime}}{t} y^{\prime}(t)+\frac{\beta^{\prime}}{t^{2}} y(t)=0
$$

with $\alpha^{\prime}=\lambda \alpha-\lambda+1$ and $\beta^{\prime}=\beta \lambda^{2}$ and with difference of indicial roots

$$
r^{\prime}=\sqrt{ }\left(\left(\alpha^{\prime}-1\right)^{2}-4 \beta^{\prime}\right)=\lambda r \text {. }
$$


Second, lift (6) to $\mathbb{C}$ by the meromorphic substitution $t=t(w)$ to obtain

$$
Y^{\prime \prime}(w)+P(w) Y^{\prime}(w)+Q(w) Y(w)=0
$$

where $P(w)=\left(-t^{\prime \prime} / t^{\prime}\right)+\alpha^{\prime}\left(t^{\prime} / t\right)$ and $Q(w)=\beta^{\prime}\left(t^{\prime} / t\right)^{2}$. Note that $\mathscr{P}$ and $\sigma$ in (2) and (3) can belong to an arbitrary lattice $L$.

Since $t=t(w)$ is a meromorphic function on $\mathbb{C}$, the transformation [6]

$$
Y(w)=e^{-1 / 2 \int^{w} P(s) d s} y(w)
$$

can be used to transform (8) into

where

$$
y^{\prime \prime}(w)+J(w) y(w)=0
$$

$$
\begin{aligned}
J(w)=Q(w)-\frac{1}{2} P^{\prime}(w)-\frac{1}{4} P^{2}(w) & =\beta^{\prime}\left(\frac{t^{\prime}}{t}\right)^{2}-\frac{1}{2}\left(-\frac{t^{\prime \prime}}{t^{\prime}}+\alpha^{\prime} \frac{t^{\prime}}{t}\right)^{\prime}-\frac{1}{4}\left(-\frac{t^{\prime \prime}}{t^{\prime}}+\alpha^{\prime} \frac{t^{\prime}}{t}\right)^{2} \\
& =\left(\beta^{\prime}+\frac{\alpha^{\prime}}{2}-\frac{\left(\alpha^{\prime}\right)^{2}}{4}\right)\left(\frac{t^{\prime}}{t}\right)^{2}+\frac{\theta_{2} t(w)}{2}
\end{aligned}
$$

Elementary calculations using (7) produce $\left(1-(\lambda r)^{2}\right) / 4=\beta^{\prime}+\left(\alpha^{\prime} / 2\right)-\left(\left(\alpha^{\prime}\right)^{2} / 4\right)$ so that

$$
J(w)=\frac{1}{2}\left[\frac{1-(\lambda r)^{2}}{2}\left(\frac{t^{\prime}}{t}\right)^{2}+\theta_{2} t(w)\right] .
$$

Equations (8) and (9) have the same ratio of linearly independent solutions.

When (2) is used for $t(w)$, we obtain [11]

$$
\left(\frac{t^{\prime}}{t}\right)^{2}=\mathscr{P}(w)-e_{3} \quad \text { and } \quad \theta_{2} t(w)=-\frac{3}{2} \mathscr{P}\left(w-\frac{\omega_{1}+\omega_{2}}{2}\right)
$$

Hence, (9) assumes the form (4) which clearly has doubly periodic coefficients.

Alternatively, when (3) is used for $t(w)$, we obtain [12]

$$
\frac{t^{\prime}}{t}=\sum_{i=1}^{k} m_{i} \zeta\left(w-a_{i}\right)-\sum_{j=1}^{1} n_{i} \zeta\left(w-b_{i}\right)+c .
$$

Hence, (9) assumes the form (5). We observe that $t^{\prime} / t$ is doubly periodic since $\sum_{i=1}^{k} m_{i}-$ $\sum_{j=1}^{1} n_{j}=0$. Also, $\theta_{2} t(w)$ is doubly periodic since $t(w)$ can be viewed as a multiplicative multi-valued function on $\mathbb{C} / L$ for some character $[3]$.

From the double periodicity of the coefficients of (4) and (5), we can conclude that these equations live on $\mathbb{C} / L$ and have monodromy groups there.

REMARK 2. The proof of this theorem implies that if the substitutions $z=t^{\lambda}(w)$ and $z=t(w), t(w)$ fixed and of form (2) or (3), are made respectively into any two Euler equations with respective differences of indicial roots $r$ and $r^{\prime}=r \lambda$, then the same transformed lifted Euler equation results. 
We are now in a position to state

THEOREM 2. Equation (4) has monodromy group $G^{*}$ given by

(a) $D_{2 n}$ if $r \lambda=k / n, n \in Z^{+}, k \in\{ \pm 1, \pm 3, \ldots\}$ and $(|k|, n)=1$

(b) $D_{n}$ if $r \lambda=k / n, n \in Z^{+}, k \in\{ \pm 2, \pm 4, \ldots\}$ and $(|k|, n)=1$

(c) $C_{2} * C_{2}$ if $r \lambda \in(\mathbb{C}-Q) \cup\{0\}$.

Remark 3. $G^{*}$ is the Klein 4-group if $n=1$ and $k \in\{ \pm 1, \pm 3, \ldots\}$ while $G^{*}$ is $C_{2}$ if $n=1$ and $k \in\{ \pm 2, \pm 4, \ldots\}$.

Proof. Remark 1 implies that there exists an equation (1) which lifts by map (2) to an equation which transforms into (4). Therefore, $\lambda=1$ can be assumed with no loss of generality. Let $u(z)$ be some ratio of linearly independent solutions to (1) and $h(w)=$ $u \circ t(w)$ the corresponding ratio of linearly independent solutions to (4). When viewed as an equation on $\mathbb{C} / L,(4)$ has singularities only at the natural projections of 0 and $\left(\omega_{1}+\omega_{2}\right) / 2$ to $\mathbb{C} / L$. Let $\Lambda_{0}^{\prime}$ and $\Lambda_{\left(\omega_{1}+\omega_{2}\right) / 2}^{\prime}$ be simple loops starting at some fixed point and enclosing the respective natural projections of 0 and $\left(\omega_{1}+\omega_{2}\right) / 2$. Let $\Lambda_{\omega_{1}}^{\prime}$ and $\Lambda_{\omega_{2}}^{\prime}$ be non-contractable loops (based at the same fixed point as above) which together generate the homotopy group of $\mathbb{C} / L$. Here, all loops avoid singularities of (4). Lift [10] $\Lambda_{0}^{\prime}$, $\Lambda_{\left(\omega_{1}+\omega_{2}\right) / 2}^{\prime}$ and $\Lambda_{\omega_{1}}^{\prime}, \Lambda_{\omega_{2}}^{\prime}$ to $\mathbb{C}$ to unique loops $\Lambda_{0}, \Lambda_{\left(\omega_{1}+\omega_{2}\right) / 2}$ and unique $\operatorname{arcs} \Lambda_{\omega_{1}}, \Lambda_{\omega_{2}}$, all having the same initial point. We can assume that the terminal points of $\Lambda_{\omega_{i}}(i=1,2)$ are translates of the common initial point by $\omega_{i}(i=1,2)$. $G^{*}$ is generated by the monodromy elements corresponding to the above loops and arcs (on $\mathbb{C}$ ).

Since $t(0)=\infty$ and $t\left(\left(\omega_{1}+\omega_{2}\right) / 2\right)=\left(\sqrt{ }\left(e_{3}-e_{1}\right)+\sqrt{ }\left(e_{3}-e_{2}\right)\right) / \sqrt{ }\left(e_{2}-e_{1}\right)=K \neq 0[\mathbf{1 1}]$, we can find [7] the generators for $G^{*}$ associated with $\Lambda_{0}$ and $\Lambda_{\left(\omega_{1}+\omega_{2}\right) / 2}$ by continuing

$$
u(z)=\left\{\begin{array}{lll}
z^{r} & \text { if } & r \in \mathbb{C}^{*} \\
\ln z & \text { if } & r=0
\end{array}\right.
$$

along the loops $\Gamma_{\infty}=t\left(\Lambda_{0}\right)$ and $\Gamma_{K}=t\left(\Lambda_{\left(\omega_{1}+\omega_{2}\right) / 2}\right)$ where $\Gamma_{\infty}$ is homotopic in $\hat{\mathbb{C}}-\{0, \infty\}$ to a simple loop enclosing $\infty$ while $\Gamma_{K}$ is homotopically trivial in this space. Thus, the generator $T_{K}$ corresponding to $\Lambda_{\left(\omega_{1}+\omega_{2}\right) / 2}$ or $\Gamma_{K}$ is the identity. Furthermore, the generator $T_{\infty}$ corresponding to $\Lambda_{0}$ or $\Gamma_{\infty}$ is

$$
T_{\infty}(z)=\left\{\begin{array}{lll}
e^{-2 \pi i r} z & \text { if } & r \in \mathbb{C}^{*} \\
z-2 \pi i & \text { if } & r=0 .
\end{array}\right.
$$

Note that $t\left(w+\omega_{1}\right)=1 / t(w)$ and $t\left(w+\omega_{2}\right)=-1 / t(w)[\mathbf{1 1}]$. Hence, we determine the generators $T_{\omega_{i}}(i=1,2)$ for $G^{*}$ arising from $\Lambda_{\omega_{i}}(i=1,2)$ by continuing $(10)$ along the arcs $\Gamma_{\omega_{\mathrm{i}}}=t\left(\Lambda_{\omega_{\mathrm{i}}}\right)$ as follows:

$$
T_{\omega_{1}}(z)=\left\{\begin{array}{lll}
\frac{e^{2 l_{1}, \pi i r}}{z} & \text { if } & r \in \mathbb{C}^{*} \\
2 l_{1} \pi i-z & \text { if } & r=0
\end{array}\right.
$$


and

$$
T_{\omega_{2}}(z)=\left\{\begin{array}{lll}
\frac{e^{\left(2 l_{2}+1\right) \pi i r}}{z} & \text { if } & r \in \mathbb{C}^{*} \\
\left(2 l_{2}+1\right) \pi i-z & \text { if } & r=0
\end{array}\right.
$$

where $l_{i} \in Z(i=1,2)$ are fixed and depend respectively on $\Lambda_{\omega_{1}}$ and $\Lambda_{\omega_{2}}$.

Replace $T_{\omega_{1}}, T_{\omega_{2}}$ and $T_{\infty}$ by the new generators [9] for $G^{*}$ given as

$$
A(z)=T_{\infty}^{l} \circ T_{\omega_{1}}(z)=\left\{\begin{array}{lll}
\frac{1}{z} & \text { if } & r \in \mathbb{C}^{*} \\
-z & \text { if } & r=0
\end{array}\right.
$$

and

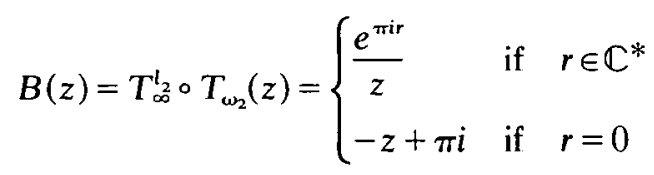

observing that $T_{\infty}$ is not needed as a generator since $T_{\infty}=(A \circ B)^{2}$.

At this point, the proof splits naturally into three cases depending on the value of $r$.

Case 1. $r=k / n, n \in Z^{+}, k \in\{ \pm 1, \pm 3, \ldots\}$ and $(|k|, n)=1$ : Let $C_{1}=A$ and $C_{2}=B \circ A$ with $A(z)$ as above and $C_{2}(z)=e^{\pi i(k / n)} z$. Clearly, $C_{1}^{2}=$ id and $C_{1} C_{2} C_{1}=C_{2}^{-1}$. Also, $C_{2}^{2 n}=$ id where elementary calculations using the oddness of $k$ show that no lower positive power of $C_{2}$ is the identity. Therefore, $G^{*}=D_{2 n}$. Hence, (a) has been verified.

Case 2. $r=k / n, n \in Z^{+}, k \in\{ \pm 2, \pm 4, \ldots\}$ and $(|k|, n)=1$ : Let $C_{1}$ and $C_{2}$ be defined as in Case 1. As above, $C_{1}^{2}=$ id and $C_{1} C_{2} C_{1}=C_{2}^{-1}$. However, $C_{2}^{n}=$ id where elementary calculations using the evenness of $k$ show that no lower positive power of $C_{2}$ is the identity. Therefore, $G^{*}=D_{n}$. Hence, (b) has been verified.

Case 3. $r \in(\mathbb{C}-Q) \cup\{0\}: A$ and $B$ satisfy $A^{2}=B^{2}=$ id. Consequently, all elements of $G^{*}$ other than $A, B$ and the identity can be realized as $(B \circ A)^{n}$ and $(B \circ A)^{n} \circ B$ for $n \in Z-\{0\}$. None of these elements is the identity for the given $r$ values. Therefore, $G^{*}=\left\langle A ; A^{2}\right\rangle *\left\langle B ; B^{2}\right\rangle$. Hence, (c) has been verified.

COROLl_ARY. The monodromy group $G^{*}$ of equation (4) is Kleinian iff $r \lambda \in$ $(\mathbb{C}-\mathrm{Re}) \cup Q$.

Proof. As in the proof of Theorem 2, we can assume that $\lambda=1$ so that $G^{*}$ is generated by

$$
A(z)=\left\{\begin{array}{lll}
\frac{1}{z} & \text { if } & r \in \mathbb{C}^{*} \\
-z & \text { if } & r=0
\end{array}\right.
$$

and

$$
B(z)=\left\{\begin{array}{lll}
\frac{e^{i \pi r}}{z} & \text { if } & r \in \mathbb{C}^{*} \\
-z+\pi i & \text { if } & r=0
\end{array}\right.
$$


Case 1. $r \in Q-\{0\}$ : By Theorem 2, $G^{*}=D_{n}$ for some $n \in Z^{+}$. Since $G^{*}$ is finite, it is Kleinian.

Case 2. $r=0: G^{*}$ is generated by $(B \circ A)(z)=z+\pi i$ and $A(z)=-z$ and therefore has fundamental domain [3]

$$
\mathrm{FD}=\{z: \operatorname{Re} z>0 \text { and } 0<\operatorname{Im} z<\pi\} .
$$

Hence, $G^{*}$ is Kleinian.

Case 3. $r \in \mathbb{C}-\operatorname{Re}$ : Now, $r=a+i b, a, b \in \operatorname{Re}, b \neq 0$ and $G^{*}$ is generated by $A(z)=$ $1 / z$ and $B(z)=e^{i \pi r} / z=e^{i \pi a-\pi b} / z$. Therefore, $a \in(0,2]$ and $b>0$ can be assumed with no loss of generality.

We will show that

$$
D=\left\{z: e^{-\pi b}<|z|<1, \quad 0<\operatorname{Arg} z<\phi\right\}
$$

(with $\phi$ determined below) is an open subset of a fundamental domain for $G^{*}$ by examining the action of the nontrivial elements of $G^{*}$ on $D$. As in Case 3 in the proof of Theorem 2, we can list all elements of $G^{*}$ as follows:

(i) id

(ii) $A$

(iii) $B$

(iv) $(B A)^{n}, n= \pm 1, \pm 2, \ldots$

(v) $(B A)^{n} B, n=+1, \pm 2, \pm 3, \ldots$.

Here, $(B A)^{n}(z)=e^{i \pi a n-\pi n b} z$ and $(B A)^{n} B(z)=e^{(i \pi a-\pi b)(1+n)} / z$.

If $T \in G^{*}$ assumes forms (ii), (iv) or (v), then

$$
\begin{aligned}
& T(D)=\left\{z: 1<|z|<e^{\pi b},-\phi<\operatorname{Arg} z<0\right\} \\
& T(D)=\left\{z: e^{-\pi b(n+1)}<|z|<e^{-\pi b n}, \pi a n<\operatorname{Arg} z<\phi+\pi a n, n \in\{ \pm 1, \pm 2, \ldots\}\right\} \\
& T(D)=\left\{z: e^{-\pi b(n+1)}<|z|<e^{-\pi b n},-\phi+\pi \dot{a}(1+n)<\operatorname{Arg} z<\pi a(1+n), n \in\{+1, \pm 2, \pm 3, \ldots\}\right\}
\end{aligned}
$$

respectively. Thus, in each instance $T(D) \cap D=\varnothing$ independently of the selection of $\phi>0$. If $T \in G^{*}$ assumes form (iii) and if $\phi=\pi a / 2$, then

$$
D=\left\{z: e^{-\pi b}<|z|<1,0<\operatorname{Arg} z<\frac{\pi a}{2}\right\}
$$

and

$$
T(D)=\left\{z: e^{-\pi b}<|z|<1, \frac{\pi a}{2}<\operatorname{Arg} z<\pi a\right\} .
$$

$T(D) \cap D=\varnothing$ since $a \in(0,2]$. Therefore, $T(D) \cap D \neq \varnothing$ only if $T$ assumes form (i). Hence, $D$ is an open subset of a fundamental domain for $G^{*}$ and $G^{*}$ is Kleinian.

Case 4. $r \in \operatorname{Re}-Q: G^{*}$ contains $(B \circ A)(z)=e^{i \pi r} z$ which is an elliptic element of infinite order since $r$ is irrational. Thus, $G^{*}$ is not Kleinian [8].

THEOREM 3. Each equation of form (5) has monodromy group $G^{*}$ of one of the following types:

$$
C_{l}, C_{\infty} \times C_{l}, C_{\infty} \times C_{\infty} \times C_{l} \quad \text { where } \quad l \in Z^{+} \cup\{+\infty\} .
$$


All of these groups (for all $l \in Z^{+} \cup\{+\infty\}$ ) are realized as $r \lambda \in \mathbb{C}$ and $t(w)$ of form (3) both vary.

Proof. Equation (5) has singularities at $a_{i}(i=1, \ldots, k), b_{j}(j=1, \ldots, l)$ and $c_{p}$ $(p=1, \ldots, t)$, the poles of $\theta_{2} t(w)$, as well as at all translates of these points by elements of the lattice $L$. Therefore, $G^{*}$ is generated by the elements corresponding to simple loops $\Lambda_{a_{i}}(i=1, \ldots, k), \Lambda_{b_{i}}(j=1, \ldots, l), \Lambda_{c_{p}}(p=1, \ldots, t)$ about the points $a_{i}, b_{j}, c_{p}$ respectively as well as arcs $\Lambda_{\omega_{i}}(i=1,2)$ defined as in the proof of Theorem 2. Since $t\left(a_{i}\right)=0$, $t\left(b_{\mathrm{j}}\right)=\infty$ and $t\left(c_{\mathrm{p}}\right)=C_{\mathrm{p}} \neq 0, \infty$, the monodromy elements $T_{a_{\mathrm{i}}}, T_{b_{\mathrm{j}}}, T_{c_{\mathrm{p}}}$ associated with the loops mentioned can be found [7] by continuing $u(z)$ in (10) along the closed loops $\Gamma_{0}^{m_{i}}=t\left(\Lambda_{a_{i}}\right)(i=1, \ldots, k), \Gamma_{\infty}^{n_{i}}=t\left(\Lambda_{b_{i}}\right)(j=1, \ldots, l)$ and $\Gamma_{C_{p}^{p}}^{O_{p}}=t\left(\Lambda_{c_{p}}\right)(p=1, \ldots, t), O_{p} \in Z$, where $\Gamma_{0}$ is homotopic in $\hat{C}-\{0, \infty\}$ to a simple loop enclosing 0 and $\Gamma_{\infty}$ is defined similarly while $\Gamma_{C_{\mathrm{n}}}$ is homotopically trivial in $\hat{\mathbb{C}}-\{0, \infty\}$. Thus, the generators $T_{c_{\mathrm{p}}}$ corresponding to $\Lambda_{c_{\mathrm{p}}}$ or $\Gamma_{C_{\mathrm{p}}^{\mathrm{n}}}^{O_{p}}$ must all be the identity. Furthermore, the generators $T_{a_{i}}$ corresponding to $\Lambda_{a_{\mathrm{i}}}$ or $\Gamma_{0}^{m_{i}}$ and the generators $T_{b_{i}}$ corresponding to $\Lambda_{b_{j}}$ or $\Gamma_{\infty}^{n_{i}}$ are respectively given by

and

$$
T_{a_{i}}(z)=\left\{\begin{array}{lll}
e^{2 \pi i m_{i} r} z & \text { if } & r \in \mathbb{C}^{*} \\
z+2 \pi i m_{i} & \text { if } & r=0
\end{array} \quad(i=1, \ldots, k)\right.
$$

$$
T_{b_{i}}(z)=\left\{\begin{array}{lll}
e^{-2 \pi i n_{i} r} z & \text { if } & r \in \mathbb{C}^{*} \\
z-2 \pi i n_{j} & \text { if } & r=0
\end{array} \quad(j=1, \ldots, l) .\right.
$$

We now show that the subgroup of $G^{*}$ generated by $T_{a_{i}}$ and $T_{b_{1}}$ can be generated by precisely one transformation $T_{0}$. We let $d_{i}$ and $e_{i}$ be integers satisfying

$$
\sum_{i=1}^{k} d_{i} m_{i}-\sum_{i=1}^{l} e_{j} n_{j}=\operatorname{gcd}\left(m_{i}, n_{j} ; \begin{array}{c}
i=1, \ldots, k \\
j=1, \ldots, l
\end{array}\right) \text {. }
$$

Define

$$
T_{0}(z)=\prod_{i=1}^{k} T_{a_{i}}^{d_{i}} \prod_{i=1}^{l} T_{b_{i}}^{e_{i}}(z)=\left\{\begin{array}{lll}
e^{2 \pi i r \operatorname{gcd}\left(m_{i}, n_{i}\right)} z & \text { if } & r \in \mathbb{C}^{*} \\
z+2 \pi i \operatorname{gcd}\left(m_{i}, n_{j}\right) & \text { if } & r=0 .
\end{array}\right.
$$

Since there exist integers $M_{i}=m_{i} / \operatorname{gcd}\left(m_{i}, n_{j}\right)$ and $N_{j}=n_{j} / \operatorname{gcd}\left(m_{i}, n_{j}\right)$, we conclude that $\left(T_{0}\right)^{M_{i}}=T_{a_{i}}(i=1, \ldots, k)$ and $\left(T_{0}\right)^{N_{i}}=T_{b_{j}}(j=1, \ldots, l)$. Therefore, $T_{0}$ generates this subgroup of $G^{*}$ as claimed.

We can view any map $z=t(w)$ of form (3) as a multi-valued function on $\mathbb{C} / L$ belonging to a multiplicative character $[3]$ generated by constants $A_{i} \in \mathbb{C}^{*}(i=1,2)$ which are associated with the natural projections of the arcs $\Lambda_{\omega_{i}}(i=1,2)$ in $\mathbb{C}$ to closed, non-contractable loops on $\mathbb{C} / L$. Equivalently, we note that $t(w)$ is a single-valued function on $\mathbb{C}$ with transformation laws $t\left(w+\omega_{i}\right)=A_{i} t(w)$ for all $w \in \mathbb{C}(i=1,2)$. Hence, the generators $T_{\omega_{i}}(i=1,2)$ corresponding to $\Lambda_{\omega_{i}}(i=1,2)$ are found by continuing $u(z)$ in (10) along the $\operatorname{arcs} \Gamma_{\omega_{i}}=t\left(\Lambda_{\omega_{i}}\right)$, where $\Gamma_{\omega_{i}}$ is closed iff $A_{i}=1$. We obtain

$$
T_{\omega_{i}}(z)=\left\{\begin{array}{lll}
A_{i}^{r} e^{2 \pi i r k} k_{i} & \text { if } & r \in \mathbb{C}^{*} \\
z+\ln A_{i}+2 \pi i k_{i} & \text { if } & r=0
\end{array} \quad(i=1,2)\right.
$$


where $A_{i}^{r}$ or In $A_{i}$ are arbitrary but fixed branches and where $k_{i} \in Z$ depend on both $\Lambda_{\omega_{i}}$ and these branches.

$G^{*}$ is generated by $T_{0}, T_{\omega_{i}}(i=1,2)$ and is a group of affine mappings consisting entirely of multiplications if $r \in \mathbb{C}^{*}$ or of translations if $r=0$. Hence, $G^{*}$ is Abelian and a direct product of at most three cyclic groups [5].

We now show that $G^{*}$ has at most one generator of finite order. If $r=0$, then clearly $G^{*}$ has no generators or nontrivial elements of finite order. If $r \in \mathbb{C}^{*}$, then assume that

$$
C_{1}(z)=e^{2 \pi i(K / M)} z, \quad C_{2}(z)=e^{2 \pi i(L / N)} z ; \quad K, L, M, N \in Z^{*}
$$

are generators of $G^{*}$ having finite order. Define

$$
D(z)=e^{(2 \pi i / M N) \operatorname{gcd}(K N . L M)} z .
$$

Number theory shows that the subgroups of $G^{*}$ generated by $C_{i}(i=1,2)$ and $D$ are the same. We conclude that $G^{*}$ has at most one generator of finite order. Therefore, $G^{*}$ is one of the types claimed.

We now show that all of these types are realized as follows:

(A) $C_{\infty} \times C_{\infty} \times C_{\infty}$ : Let $r=0$ and

$$
t(w)=\left(\sigma\left(w-\frac{\omega_{1} \sqrt{ } 2-\omega_{2}}{2 \pi i}\right) / \sigma(w)\right) \cdot e^{\left(\left(n_{1} \sqrt{ } 2-n_{2}\right) / \pi i\right) w}
$$

where $\eta_{i}=\zeta\left(\omega_{i} / 2\right)(i=1,2)$. Then, $A_{1}=e, A_{2}=e^{\sqrt{2}}$ and $\operatorname{gcd}\left(m_{i}, n_{i}\right)=1$. Thus, $G^{*}$ is generated by

$$
\left\{\begin{array}{l}
T_{0}(z)=z+2 \pi i \\
T_{\omega_{1}}(z)=z+1+2 \pi i k_{1} \\
T_{\omega_{2}}(z)=z+\sqrt{ } 2+2 \pi i k_{2}, \quad k_{i} \in Z \quad(i=1,2)
\end{array}\right.
$$

where the cyclic subgroups generated by $T_{0}, T_{\omega_{1}}$ and $T_{\omega_{2}}$ are disjoint. Hence, $G^{*}=$ $C_{\infty} \times C_{\infty} \times C_{\infty}$.

(B) $C_{\infty} \times C_{\infty} \times C_{l}$, for all $l \in Z^{+}$: Let

$$
r=\frac{1}{l} \text { and } t(w)=\left(\sigma\left(w-\frac{\omega_{1} \ln 3-\omega_{2} \ln 2}{2 \pi i}\right) / \sigma(w)\right) \cdot e^{\left(\left(\eta_{1} \ln 3-n_{2} \ln 2\right) / \pi i\right) w} .
$$

Here, $A_{1}=2, A_{2}=3$ and $\operatorname{gcd}\left(m_{i}, n_{j}\right)=1$. Thus, $G^{*}$ is generated by

$$
\left\{\begin{array}{l}
T_{0}(z)=e^{2 \pi i / l} z \\
T_{\omega_{1}}(z)=2^{1 / /} e^{(2 \pi i /) k_{1}} z \\
T_{\omega_{2}}(z)=3^{1 / l} e^{(2 \pi i / l) k_{2}} z, \quad k_{i} \in Z \quad(i=1,2) \quad \text { and } 2^{1 / 1}, 3^{1 / /} \in \mathrm{Re}^{+} .
\end{array}\right.
$$

The disjoint cyclic subgroups generated by $T_{\omega_{1}}$ and $T_{\omega_{2}}$ are of infinite order and are both disjoint from the cyclic subgroup of order $l$ generated by $T_{0}$. Hence, $G^{*}=C_{\infty} \times C_{\infty} \times C_{l}$ where $l \in Z^{+}$is arbitrary. Note that when $l=1$, we obtain $G^{*} C_{\infty}=C_{\infty}$. 
(C) $C_{\infty} \times C_{l}$, for all $l \in Z^{+}$: Let

$$
r=\frac{1}{l} \quad \text { and } \quad t(w)=\left(\sigma\left(w-\frac{\omega_{1} \ln 3}{2 \pi i}\right) / \sigma(w)\right) \cdot e^{((\eta, \ln 3) / \pi i) w} .
$$

Here, $A_{1}=1, A_{2}=3$ and $\operatorname{gcd}\left(m_{i}, n_{i}\right)=1$. Thus, $G^{*}$ is generated by

$$
\left\{\begin{array}{l}
T_{0}(z)=e^{2 \pi i / l} z \\
T_{\omega_{1}}(z)=e^{2 \pi i\left(k_{1} l l\right)} z \\
T_{\omega_{2}}(z)=3^{1 / l} e^{2 \pi i\left(k_{2} l l\right)} z, \quad k_{i} \in Z \quad(i=1,2) \quad \text { and } \quad 3^{1 / l} \in \mathrm{Re}^{+}
\end{array}\right.
$$

$T_{\omega_{1}}=T_{0}^{k}, O\left(T_{0}\right)=l$ and $O\left(T_{\omega_{2}}\right)=\infty$ imply that $G^{*}=C_{\infty} \times C_{l}$. Note that when $l=1$, we obtain $G^{*}=C_{\infty}$.

(D) $C_{l}$, for all $l \in Z^{+}$: Let

$$
r=\frac{1}{l} \quad \text { and } \quad t(w)=\left(\sigma^{2}\left(w-\frac{\omega_{1}+\omega_{2}}{4}\right) / \sigma\left(w-\frac{\omega_{1}}{2}\right) \sigma\left(w-\frac{\omega_{2}}{2}\right)\right) .
$$

Here, $A_{i}=1(i=1,2)$ and $\operatorname{gcd}\left(m_{i}, n_{j}\right)=1$. Thus, $G^{*}$ is generated by

$$
\left\{\begin{array}{l}
T_{0}(z)=e^{2 \pi i / l} z \\
T_{\omega_{i}}(z)=e^{2 \pi i\left(k_{i} / l\right)} z, \quad k_{i} \in Z \quad(i=1,2) .
\end{array}\right.
$$

Since $T_{\omega_{\mathrm{i}}}=\left(T_{0}\right)^{k_{i}}$ and $O\left(T_{0}\right)=l$, we conclude that $G^{*}=C_{l}$. Note that when $l=1, G^{*}=i d$.

Remark 4. If $t(w)$ of form (3) is doubly periodic (or equivalently if $A_{i}=1$ for $i=1,2)$, then the $\operatorname{arcs} \Lambda_{\omega_{i}}(i=1,2)$ as well as all loops $\Lambda_{a_{i}}$ and $\Lambda_{b_{i}}$ project under $t$ to closed loops about 0 (or $\infty$ ) in $\ddot{\mathbb{C}}-\{0, \infty\}$. Thus, $G^{*}$ is a (cyclic) subgroup of the group $G$ of (1) when $\lambda=1$ and $t$ is as above.

In fact, we can prove

THEOREM 4. The group $G^{*}$ of the equation (5) obtained by lifting an arbitrary equation (1) by any non-constant doubly periodic function $t(w)(\lambda=1)$ is a proper subgroup of the monodromy group $G$ of (1) if

(I) $1 \neq \operatorname{gcd}\left(O(G), m_{i}, n_{j} ; \begin{array}{c}i=1, \ldots, k \\ j=1, \ldots, l\end{array}\right)=M_{1}$ when $O(G)$ is finite or

$$
1 \neq \operatorname{gcd}\left(m_{i}, n_{i} ; \begin{array}{c}
i=1, \ldots, k \\
j=1, \ldots, l
\end{array}\right)=M_{2} \text { when } O(G)=\infty
$$

and if

(II) there exists a doubly periodic root function $t^{1 / M_{2}(w) . ~}$

In fact, if (I) and (II) are satisfied, then $\left[G: G^{*}\right]=M_{1}$ or $M_{2}$ depending respectively on $O(G) \in Z^{+}$or $O(G)=\infty$.

Remark 5. For a given monodromy group $G$, mappings $t$ can be constructed for which $\left[G: G^{*}\right]$ in $Z^{+}$is arbitrary. Thus, every subgroup of $G$ is the monodromy group of an equation lifted from (1) by some map $t$. 
Proof. The existence of a doubly periodic root function $t^{1 / M_{2}(w)}$ implies that $t\left(\Lambda_{\omega_{i}}\right)=$

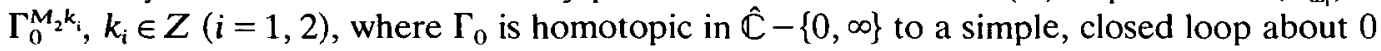
[7]. Therefore, the generators $T_{\omega_{i}}(i=1,2)$ are given by

$$
T_{\omega_{1}}(z)=\left\{\begin{array}{lll}
e^{2 \pi i r M_{2} k_{i}} z & \text { if } & r \in \mathbb{C}^{*} \\
z+2 \pi i M_{2} k_{i} & \text { if } & r=0
\end{array} \quad(i=1,2) .\right.
$$

Since $T_{\omega_{i}}=\left(T_{0}\right)^{k_{i}}$ where $T_{0}$ is defined as in Theorem 3 by

$$
T_{0}(z)=\left\{\begin{array}{lll}
e^{2 \pi i r M_{2} z} & \text { if } & r \in \mathbb{C}^{*} \\
z+2 \pi i M_{2} & \text { if } & r=0
\end{array}\right.
$$

it follows that $G^{*}$ is generated by $T_{0}$ alone.

Furthermore, $G$ is generated by

$$
D_{0}(z)=\left\{\begin{array}{lll}
e^{2 \pi i r} z & \text { if } & r \in \mathbb{C}^{*} \\
z+2 \pi i & \text { if } & r=0 .
\end{array}\right.
$$

We now determine the relationship between $G^{*}$ and $G$ by consideration of the following two cases.

Case 1. If $r \in(\mathbb{C}-Q) \cup\{0\}$, then $G=\left\langle D_{0}\right\rangle$ is free and $G^{*}=\left\langle T_{0}\right\rangle=\left\langle D_{0}^{M_{2}}\right\rangle$ is a free proper subgroup of index $M_{2}$ in $G$. We note that both $G^{*}$ and $G$ are purely parabolic, elliptic or loxodromic.

Case 2. If $r \in Q-\{0\}$, then $O(G) \in Z^{+}$and $r=l / O(G)$ where $l \in Z-\{0\}$ and $\operatorname{gcd}(|l|$, $O(G))=1$. Note that $O(G) \neq 1$. Otherwise, hypothesis I would be contradicted. Therefore, $r \notin Z-\{0\}$ and $G=\left\langle D_{0} ; D_{0}^{O(G)}=1\right\rangle$ is not trivial. Also, as in Case 1, $G^{*}$ is generated by $T_{0}=D_{0}^{\mathrm{M}_{2}}$.

We now determine $\left[G: G^{*}\right]$ and show that $G^{*}$ is a proper subgroup of $G$. Observe that $G^{*}=\left\langle D_{0}^{M_{2}} ; D_{0}^{O(G)}=1\right\rangle$. Since $M_{1}=\operatorname{gcd}\left(O(G), M_{2}\right)$, there exist $k_{1}, k_{2} \in Z$ such that $k_{1} O(G)+k_{2} M_{2}=M_{1}$. Thus, $D_{0}^{M_{1}}=D_{0}^{k_{1} O(G)+k_{2} M_{2}}=D_{0}^{k_{2} M_{2}}$ is an element of $G^{*}$ which must generate $G^{*}$ since $M_{1} \mid M_{2}$. Hence, $G^{*}=\left\langle D_{0}^{M_{1}} ; D_{0}^{O(G)}=1\right\rangle$. Suppose that there exists $L \in Z-\{0\}$ such that $|L|<M_{1}$ with $D_{0}^{L} \in G^{*}$. Since $D_{0}^{M_{2}}$ generates $G^{*}$, there exists $k_{3} \in Z-\{0\}$ with $D_{0}^{L}=D_{0}^{k_{3} M_{2}}$. It follows that $L \equiv k_{3} M_{2} \bmod O(G)$ and there exists $k_{4} \in Z$ with $L=k_{3} M_{2}+k_{4} O(G)$. This statement is contradicted by the fact that $M_{1}=$ $\operatorname{gcd}\left(O(G), M_{2}\right)$. Therefore, if $D_{0}^{L} \in G^{*}$, then $M_{1} \mid L$. This result together with the facts that $G^{*}=\left\langle D_{0}^{M} ; D_{0}^{O(G)}=1\right\rangle$ and $M_{1} \mid O(G)$ imply, by group theory, that $O\left(G^{*}\right)=O(G) / M_{1}$. Hence, $\left[G: G^{*}\right]=M_{1}$. Since $M_{1}>1, G^{*}$ is a proper subgroup of $G$.

\section{REFERENCES}

1. W. E. Boyce and R. C. DiPrima, Elementary differential equations and boundary value problems (Wiley 1977).

2. A. Erdélyi et al., Higher transcendental functions (Bateman Manuscript Project, Vol. 1 (McGraw-Hill, 1953).

3. H. Farkas and I. Kra, Riemann surfaces (Springer-Verlag, 1980). 
4. R. C. Gunning, Special coordinate coverings of Riemann surfaces, Math. Annalen, 170, (1967) 67-86.

5. M. Hall, The theory of groups (Macmillan 1970).

6. H. Hochstadt, The functions of mathematical physics (Interscience, 1971).

7. K. Kuiken, The effect of rational substitutions on the monodromy group of the general $n$th order equation of the Fuchsian class, Math. Z., 163, (1978) 111-119.

8. J. Lehner, A short course in automorphic functions (Holt, Rinehart and Winston, 1966).

9. W. Magnus, A. Karrass and D. Solitar, Combinatorial group theory (Interscience, 1966). 1967).

10. W. S. Massey, Algebraic topology: an introduction (Harcourt, Brace and World, New York,

11. J. T. Masterson, Branched affine (or projective) structures on Riemann surfaces, $\mathrm{Ph} . \mathrm{D}$. Thesis, (1981) Polytechnic Institute of New York, (to appear).

12. R. Nevanlinna and V. Paatero, Introduction to complex analysis (Addison-Wesley, 1962).

13. H. Poincaré, Sur les groupes des équations linéaires, Acta Math., 4, (1884) 201-312.

14. E. G. C. Poole, Theory of linear differential equations (Dover, 1960).

POlyteChNic Institute of NeW YoRk, 333 JAY STREET,

BROOKLYN,

NEW YORK 11201,

U.S.A.
Seton Hall University, 435 South Orange Avenue, South Orange, New Jersey 07079, U.S.A. 